\title{
JOHN BARTH COMO PARADIGMA DE LOS PROBLEMAS QUE PLANTEA LA TRADUCCIÓN DE LA NOVELÍSTICA POSTMODERNA ESTADOUNIDENSE Javier Martín Párraga Universidad de Córdoba
}

\section{ABSTRACT}

The present paper examines the complexities inherent to the translation of American postmodern narrative. In order to do so, I have chosen to consider Postmodernism as a socio-cultural phenomenon. From the point of view of translation, in this paper I will study the difficulties that can be seen when translating John Barth's extraordinary corpus.

KEYWORDS: Translation, Postmodernism, John Barth, Deconstruction

\section{RESUMEN}

El artículo propuesto examina las complejidades inherentes a la traducción de la narrative postmoderna norteamericana. Para llevar a cabo este objetivo, se considera el Postmodernismo como fenómeno socio-cultural. Desde la perspectiva traductológica, el artículo estudia las dificultades que entraña la traducción del extraordinario corpus narrative de John Barth.

PALABRAS CLAVE: traducción, posmodernismo, John Barth, deconstrucción

\section{ALGUNAS CONSIDERACIONES PREVIAS SOBRE LA NARRATIVA POSTMODERNA}

Puesto que la novelística norteamericana de la segunda mitad del siglo $\mathrm{XX}$ supone una de las mayores cotas de excelencia artística y complejidad formal alcanzadas en la narrativa mundial, acometer la tarea de traducir un texto postmoderno o, al menos, reflexionar sobre las dificultades inherentes a dicha tarea, constituye una tarea tan excitante como compleja. Por lo tanto, estimo necesario comenzar el presente trabajo reflexionando, si bien de manera somera y modesta, sobre la propia naturaleza de esta manifestación literaria. 
En este sentido, iniciaremos nuestra tarea señalando que, a pesar de los innumerables estudios que sobre este aspecto concreto han venido proliferando a lo largo de las últimas décadas, nos encontramos aún muy lejos de haber alcanzado un mínimo consenso sobre el mismo, hecho que sin duda dificulta nuestro proyecto.

Sin duda, el primer obstáculo que plantea el estudio del postmodernismo, por no hablar de cualquier intento de trazar una definición concluyente o iniciar una aproximación de carácter taxonómico del mismo, deriva del propio término. ¿Hemos de entender que "post" implica una clasificación de tipo netamente temporal o cronológica? En otras palabras, $¿$ Resulta coherente afirmar que el postmodernismo puede ser considerado como un fenómeno artístico y socio-cultural que sucede de manera estrictamente cronológica al modernismo o, por el contrario, nos estamos situando ante una catalogación (bastante ambigua) de tipo conceptual? Si damos por válida la primera hipótesis, nos enfrentaríamos a una corriente filosófica, artística y cultural que acontece tras la explosión vanguardista, sumamente refinada, que supuso el modernismo. En caso de decantarnos por la segunda opción, el postmodernismo no vería la luz tras el modernismo, sino que "iría más allá" que éste, tanto en su dimensión moral como desde una perspectiva estética.

En cualquiera de los dos escenarios propuestos, las respuestas arrojadas resultarían tentativas en el mejor de los casos, ya que hay expertos que no dudan en clasificar determinadas obras artísticas de los años 60 del siglo veinte como modernistas, momento temporal en el que el modernismo debería llevar superado ya varias décadas, al menos de acuerdo con la mayoría de voces críticas. Por otra parte, si aceptamos que el postmodernismo va "más allá" cabría considerar si la novelística de Pynchon supera, en efecto, en cuanto a naturaleza experimental y anti-realista a Finnegans Wake, obra que James Joyce publicó en el año 1939 y que desde ese momento ha constituido una suerte de pesadilla para cualquier crítico literario o traductor que osa acercarse a la misma.

La conclusión, aunque debamos aceptar una vez más la esencial marca de provisionalidad a la que referimos con anterioridad, a la que llegamos es que ambas definiciones son parcialmente correctas, si bien evidentemente incompletas. De este modo, tras estudiar a los principales exponentes del movimiento postmoderno (como puedan ser Linda Hutcheon, Fredrerick Jameson, Michel Foucault, o Roland Barthes, por sólo citar algunos ejemplos paradigmáticos), no nos queda sino afirmar que no nos encontramos en verdad ante una pareja de opuestos binarios excluyentes, sino más bien ante dos conceptos que (si bien de manera paradójica en determinadas ocasiones) se complementan de manera simbiótica y casi parasitaria. 
En cualquier caso, resulta innegable que el postmodernismo, lleva la concepción de William Empson de la ambigüedad como semilla del texto literario a extremos en los que ésta justifica la propia estructura narrativa. En palabras de Roland Barthes,

In the multiplicity of writing, everything is to be disentangled, nothing deciphered; the structure can be follow, 'run' (like the thread of a stocking) at every point and at every level, but there is nothing beneath: the space of writing is to be ranged over, not pierced; writing ceaselessly posits meaning ceaselessly to evaporate it, carrying out a systematic exemption of meaning (2000: 145).

Así pues, el texto postmoderno se resiste en todo momento a aceptar la posibilidad de una lectura unívoca, negándole al lector el placer de efectuar una interpretación "verdadera" del texto literario, ya que éste no supone un tránsito lineal como tradicionalmente se había aseverado. Como tampoco supone un paseo por un bosque ficcional, si deseamos emplear la metáfora de Umberto Eco, sino más bien un jardín de senderos que se bifurcan, a la manera de Jorge Luis Borges.

Así pues, lejos de ofrecernos artefactos literarios cuidadosamente diseñados pero unidimensionales, el postmodernismo plantea una serie de realidades ficcionales que pueden ser calificadas como poliédricas en unas ocasiones y como auténticas hidras significativas en otras. Como ejemplo paradigmático de este tipo de estructura narrativa proponemos la novela del narrador italiano Italo Calvino, Se una notte d'inverno un viaggatore.

$\mathrm{Si}$ aceptamos como verdadera la evidente hipótesis de que el primer paso en toda traducción literaria no es sino el análisis y comprensión rigurosa del texto fuente, comenzaremos a intuir el abismo hermenéutico al que se enfrenta el traductor que tiene ante sí la protéica tarea de llevar al castellano las novelas de cualquier novelista postmoderno.

Tras expresar con brevedad la naturaleza polidimensional y esquiva de los textos postmodernos, conviene señalar las fuentes filosóficas de las que beben los mismos. Las pócimas que consume el postmodernismo para alcanzar su esencia múltiple y paradójica son tan diversas como complejas. En primer lugar, resulta innegable que las teorías de Sigmund Freud y alguno de sus discípulos aventajados como el controvertido pero no por ello menos influyente Carl Gustav Jung o Jacques Lacan resultan seminales. El psicoanálisis abría la puerta a un universo desconocido hasta el momento y que resultaba tan apasionante y desconcertante como imposible de reducir a categorías estancas e inmutables: el subconsciente. Si bien podemos sumergirnos en el estudio del "id, ego y superego" (Freud), el "inconsciente 
colectivo" o los "arquetipos universales" (Jung) o las "pulsiones" (Lacan); al final de nuestro estudio no podremos sino aceptar la incognoscibilidad de los mecanismos más profundos de la mente humana, puesto que éstos operan mediante un lenguaje que nuestra parte consciente entiende como desorganizada y caótica. Un claro ejemplo de esto lo constituyen las célebres imágenes presentadas por la Gestalt o las geometrías imposibles de Escher, que ofrecen paradojas irresolubles en las que una misma representación gráfica ofrece dos realidades representadas opuestas; aunque siempre complementarias, entrelazadas y dependientes entre sí. Una vez más, los opuestos binarios no se autoexcluyen sino que conviven en una paradójica armonía que nos atrevemos a denominar, recurriendo a las ciencias experimentales que tanto frecuentan los autores postmodernos, como entrópica.

Además del psicoanálisis, el postmodernismo se dirige a otras fuentes filosóficas en busca de inspiración y guía. Pecaríamos de űßpis si nos propusiéramos aquí el rastrear todas y cada una de las influencias filosóficas que se abren camino en el postmodernismo; pero se hace indispensable hacer referencia, al menos, a Friedritch Nietzsche y Martin Heidegger como "abuelos" y a Jacques Derrida, Michel Foucault, Jean-François Lyotard o Jean Baudrillard como alguno de los ideólogos más reseñables del movimiento. De Nietzsche se toma su análisis de los conceptos genealógicos; de Heidegger el concepto de escritura "sous rature", de Derrida destacan las ideas de la "differance" y el "pharmakon", Foucault aporta su radical escepticismo hacia las instituciones tradicionales y los conceptos como "cordura", "sanidad" o "justicia"; Lyotard contribuye al postmodernimo con la formulación de las "petit récits"; mientras que el más reciente "simulacrum" de Baudrillard no resulta menos importante para el postmodernismo.

Como confesábamos con anterioridad, no podemos, ni pretendemos explicar en este trabajo todos estos conceptos y teorías filosóficas (tarea proteica e inalcanzable en demasiados sentidos), sino simplemente apuntar que de esta amalgama de postulados filosóficos se erige un entendimiento de la realidad exterior e interior que resulta paradójica, compleja en extremo y, ante todo, múltiple. Como vemos (porqué no confesarlo, no sin cierto desconsuelo), lejos quedan ya los tiempos de las certezas y las filosofías cartesianas que aspiraban a explicar el mundo en términos racionales de cabal belleza aritmética. Como Malcolm Ruland y Roland Bradbury acertadamente señalan, la literatura del siglo XX, "demands its discovering fictions, fictions adequate to explore the strange, ever more plural world to come" (1991: 429). De este modo, la literatura postmoderna no deja de ser aristotélica en el sentido de que su aspiración profunda no es sino la mímesis de la realidad. Lo que ocurre es 
que la realidad que debe ser reflejada se entiende ahora, tras Auschwitz e Hiroshima, como caótica y desestructurada.

Por último, debemos apuntar una nueva influencia para el postmodernismo a la que, desde nuestro punto de vista, no se ha prestado hasta el momento la adecuada atención: las teorías científicas relacionadas con la física cuántica expuestas por Albert Einstein, Nathan Rosen o Erwin Schrödinger. En este sentido, ya en 1950 Leopold Infeld afirmaba que, "relativity theory, essentially the work of one man, changed our concepts of space, time, mass, energy, gravitation, and the geometry of the world" (1950: 112). Tras la celebérrima fórmula $\Sigma=m c 2$ se ocultaba una auténtica caja de Pandora filosófica de proporciones indescriptibles. En primer lugar, como Hans Reichenbach apunta "the analysis of knowledge has always been the basic issue of philosophy; and if knowledge in so fundamental a domain as that of space and time is subject to revision, the implications of such criticism will involve the whole of philosophy" (1970: 290). La física cuántica y la teoría de la relatividad permitían conocer el universo como nunca se había logrado, además de imponer una manera nueva de enfrentarse al mismo, incompatible con concepciones, dogmas y axiomas ancestrales y tradicionalmente aceptados. Desde un prisma cuántico, el universo no debe concebirse de manera lineal, sino más bien como una entidad altamente flexible que se pliega y repliega sobre sí misma, por lo que el concepto de tiempo se problematiza. Este hecho lleva a admitir una nueva dimensión ante la cual la ontológica oposición binaria entre ser/no ser deja de tener sentido. Como Stanley L. Jaki apunta "Einstein's work on relativity was not yet completed when it began to be taken for the scientific proof of the view that everything is relative. Such a view, widely entertained on the popular as well as on the academic level, is now a climate of thought" (1987: 5). De esta manera, es coherente definir a Einstein como a un "maker of modern culture", definición que le da Roland N. Stromberg (1991) en un volumen en el que estudia a los cinco pensadores que, desde su punto de vista, han contribuido mejor a cimentar el entendimiento que tenemos del mundo en el siglo XX y albores del XXI. Si la influencia de Einstein es crucial para el postmodernismo, el artículo que Erwin Schrödinger publica 1933 para ilustrar sus descubrimientos sobre la naturaleza y comportamiento de los electrones no lo es menos. En este trabajo, Schrödinger formula su célebre "paradoja del gato", mediante la cual resulta perfectamente posible (por sorprendente, paradójico o incluso descabellado que pueda resultar) hallar un escenario en el que los estados de vida y muerte se simultaneen y resulten complementarios. En otras palabras, el premio Nobel acababa de desconstruir el opuesto binario ontológico por antonomasia, "vida vs muerte". Si se acepta que la paradoja de Schrödinger está en efecto bien formulada (y en más de siete décadas la teoría no ha podido ser desmontada), se debe aceptar que, dadas 
determinadas circunstancias y a raíz de la propia estructura atómica de los electrones, un ser vivo podría llegar a estar simultáneamente vivo y muerto. Huelga decir que las implicaciones de dicha afirmación hacen tambalearse los cimientos de la filosofía tradicional e invalidan, casi por completo, postulados que han sido considerados durante centurias o incluso milenios como verdades universales. En resumidas cuentas, con su paradoja, Schrödinger desconstruye de un plumazo opuestos binarios sobre los que se cimenta la visión tradicional del mundo, al abrir la puerta a la posibilidad de existencias simultaneas pero completamente opuestas que, sin embargo, no se invalidan como, al menos en apariencia, debieran hacer.

Como resultado de los fundamentos que acabamos de reflejar, el postmodernismo acaba por desembocar en lo que Linda Hutcheon denomina como un auténtico "state of crisis in representation" (2002: 29).

\subsection{JOHN BARTH Y SUS PROBLEMAS TRADUCTOLÓGICOS}

Nos proponemos en esta sección ofrecer un ejemplo paradigmático de los problemas que presentan de cara a su traducción a otros idiomas los textos postmodernos. Si la tarea traductológica, de manera muy somera, consiste en trasladar un mensaje desde la lengua fuente hasta la de destino, ¿qué hacer ante un mensaje que ya en la lengua fuente se manifiesta como bifurcado y múltiple?; ¿cómo abordar la traducción de unos textos que presentan la posibilidad de desentrañar el significado como una aporía? De entre la miríada de textos que podrían servirnos para ilustrar esta cuestión (entre los que destacan The Crying of Lot 49, de Thomas Pynchon Slaughtehrouse-Five o Mother Night, de Kurt Vonnegut; White Noise, de Don Delillo; o, incluso novelas más recientes como Lunar Park, de Bret Easton Ellis o Fight Club, de Chuck Palahniuk), hemos optado por examinar algunos ejemplos correspondientes al corpus ficcional del narrador norteamericano John Barth.

Barth, nacido en 1930, es una de las figuras clave dentro del movimiento postmoderno, tanto gracias a su extenso corpus literario como a raíz de su dilatada experiencia como profesor universitario durante la que ha publicado cientos de artículos (entre los que brillan con luz propia los seminales "The Literature of Exhaustion" $y$ "The Literature of Replenishment", textos en los que se contempla con lucidez extrema la supuesta muerte de la novela).

En lo concerniente a su producción ficcional, Barth ha dado a la imprenta las novellas The Floating Opera (1956) y The End of the Road (1958); los libros de narrativa (muy) extensa The Sot-Wed Factor (1960); Giles Goat-Boy (1966); Chimera (1972); LETTERS (1979); Sabbatical: a Romance (1982); The 
Tidewater Tales (1987); The Last Voyage of Somebody, the Sailor (1991); Once Upon a Time: A Floating Opera (1994); Coming Soon: A Narrative (2001); The Development (2008); Every Third Thought: a Novel in Five Seasons (2011). Asimismo, también ha publicado tres excepcionales colecciones de relatos breves: Lost in the Funhouse (1968); On with the Story (1996); The Book of Ten Nights and a Night (2004).

La literatura de Barth, que puede considerarse no sólo como paradigmáticamente postmoderna sino incluso como el modelo a través del que se comparan otras obras literarias a la hora de definirlas como pertenecientes a dicho movimiento artístico, se caracteriza principalmente por los rasgos que, de manera somera, pasamos a señalar a continuación.

En primer término, como Enrique García Díez acertadamente señala, en el corpus del autor "todos los hechos pasan una y otra vez por el filtro de la palabra hasta que finalmente sólo queda un ilusionismo de verdad, palabras únicamente" (1991: 60). De este modo, el lenguaje deja de ser denotativo para transmutarse en una manifestación connotativa sumamente porosa.

Otro rasgo característico del corpus literario de John Barth viene derivado de la ilusión de historicidad y deconstrucción del opuesto binario Historia/relato que se lleva a cabo en el mismo de manera muy similar a la propuesta en los escritos filosóficos de autores como Jean François Lyotard, Michel Foucault o Jacques Derrida, a los que ya referimos con anterioridad.

Por último, no podemos dejar de señalar el interés que el autor muestra desde sus primeros escritos por las teorías psicoanalíticas y de la filosofía de la Gestalt, que también insisten en la posibilidad de la existencia de elementos mutuamente excluyentes, pero que son al mismo tiempo no sólo compatibles sino complementarios.

Como resulta evidente, si el universo literario barthiano se cimenta en una concepción de la realidad netamente lingüística que desconfía del mundo exterior hasta el punto de considerarlo mediatizado por una serie de filtros y especialmente dependiente del lenguaje que lo define/constituye, la traducción de la pléyade de obras que componen dicho universo resultará tan titánica como proteica.

Así pues, los escritos de Barth hacen gala de una estructura interna poliédrica que basa su funcionamiento en los conceptos de escritura sous rature de Heidegger y la denominada como "paradoja del gato" de Schrödinger, a los que referimos con anterioridad. De este modo, John Barth va más allá de la ambigüedad al presentar una serie de situaciones y personajes que ofrecen una interpretación dual en todo momento.

Las páginas del autor abren la puerta, pues, a una serie de cuestiones que el propio desarrollo argumental de las obras se esfuerzan en dejar irresolutas en todos y cada uno de los casos, hasta conformar una tela de araña enrevesada y borgiana en la que posibilidades antagónicas se presentan como 
simultáneamente ciertas y falsas, a la manera en que el gato de Schrödinger estaba vivo y muerto al mismo tiempo.

La pregunta que se hace la protagonista de la novela Th Crying of Lot 49, de Thomas Pynchon (1966) define a la perfección el angst de los personajes de Barth, que inseguros de hasta qué punto están contemplando la realidad o, por el contrario, son presa de su propia confusión mental se plantean: "Shall I project a world?" (1966: 76).

El problema que se plantea de cara a la traducción resulta tan evidente como desesperanzador: si la única realidad apreciable en estas novelas corresponde a la que tiene lugar en la mente de sus protagonistas (un escenario al que ni ellos misma tiene el pleno acceso garantizado) y la mente es siempre multipolar y caótica, ¿cómo puede el lector formarse una idea única de lo que está aconteciendo? En otras palabras, ¿si los personajes navegan en un océnao de dudas y ambigüedades o no, cómo puede estar seguro en ningún caso el lector? ¿Y el traductor?

\section{CONCLUSIONES}

Tras analizar con profundidad el corpus literario de John Barth, estamos firmemente convencidos de que la única opción viable a la hora de traducir su extraordinario corpus pasa necesariamente por desentrañar parte de la maraña conceptual que se establece en el mismo, transitando uno de los senderos posibles y despreciando otros. De esta manera, no se ofrece al lector la hidra que supone la novela original, sino tan sólo una de sus múltiples cabezas. No obstante, si no se acepta esta situación como un mal necesario, no queda sino aceptar la imposibilidad de traducir el texto a otros idiomas. Así pues, debemos aceptar la traducción de los textos postmodernos, con todas sus trabas y pérdida de riqueza literaria con respecto al texto fuente que presentan, de manera consecuente con el texto fuente. Es decir, la traducción de una novela postmoderna será siempre una experiencia heiddegariana de escritura y lectura "sous rature"

\section{REFERENCIAS BIBLIOGRÁFICAS}

BARTH, John. The Floating Opera. New York,: Appleton-Century-Crofts, 1956.

---. The End of the Road. [1st ed. Garden City, N.Y.: Doubleday, 1958.

---. The Sot-Weed Factor. [1st ed. Garden City, N.Y.,: Doubleday, 1960.

---. Giles Goat-Boy; or, the Revised New Syllabus. Garden City, N.Y.,: Doubleday, 1966. 
---. Lost in the Funhouse; Fiction for Print, Tape, Live Voice. [1st ed. Garden City, N.Y.,: Doubleday, 1968.

---. Chimera. [1st ed. New York,: Random House, 1972.

---. Letters : A Novel. New York: Putnam, 1979.

---. Sabbatical: A Romance. New York: Putnam, 1982.

---. The Friday Book : Essays and Other Nonfiction. New York, NY: Putnam, 1984.

---. The Tidewater Tales : A Novel. New York: Putnam, 1987.

---. The Last Voyage of Somebody the Sailor. 1st ed. Boston: Little, Brown, 1991.

---. Once Upon a Time : A Floating Opera. 1st ed. Boston: Little, Brown, 1994.

---. Further Fridays : Essays, Lectures, and Other Nonfiction, 1984-94.

Boston: Little, Brown and Co., 1995.

---. On with the Story : Stories. 1st ed. Boston: Little, Brown, 1996.

---. Coming Soon!!! : A Narrative. Boston: Houghton Mifflin, 2001.

---. The Book of Ten Nights and a Night : Eleven Stories. Boston: Houghton Mifflin, 2004.

---. Where Three Roads Meet: Novellas. Boston: Houghton Mifflin, 2005.

---. The Development: Nine Stories. Boston: Houghton Mifflin Co., 2008.

---. Every Third Thought : A Novel in Five Seasons. Berkeley, CA: Counterpoint, 2011.

BARTHES, Roland. "The Death of the Author." Falling into Theory: Conflicting Views on Reading Literature. Ed. Richter, David H. Boston: Bedford/St. Martin's, 2000.

BAUDRILLARD, Jean. Simulacres Et Simulation. Débats. Paris: Galilée, 1981.

---. La Guerre Du Golfe N’a Pas Eu Lieu. Paris: Galilée, 1991.

---. Car L'illusion Ne S'oppose Pas À La Réalité. Paris: Descartes \& Cie, 1998.

BORGES, Jorge Luis. Obras Completas. Barcelona: Emecé, 1996.

CALVINO, ITALO. Se Una Notte D'inverno Un Viaggiatore. Torino: Einaudi, 1979.

DELILLO, Don. White Noise. New York: Viking, 1985.

ECO, Umberto. Six Walks in the Fictional Woods. Cambridge: Harvard University Press, 1994.

ELLIS, Bret Easton. Lunar Park. New York: Knopf, 2005.

EMPSON, William. Seven Types of Ambiguity. London: Chatto and Windus, 1930.

FOUCAULT, Michel. Madness and Civilization; a History of Insanity in the Age of Reason. New York: Pantheon Books, 1965.

---. Surveiller Et Punir: Naissance De La Prison. Paris: Gallimard, 1975.

FREUD, Sigmund. Writings on Art and Literature. Stanford: Stanford University Press, 1997.

---. The Standard Edition of the Complete Psychological Works of Sigmund Freud. New York: Norton, 1989. 
FREUD, Sigmund, y James STRACHEY. The Complete Introductory Lectures on Psychoanalysis. London: Allen \& Unwin, 1971.

HEIDEGGER, Martin, Pathmarks. New York: Cambridge University Press, 1998.

HUTCHEON, Linda. Narcissistic Narrativ : The Metafictional Paradox. Waterloo: Wilfrid Laurier University Press, 1980.

---. A Poetics of Postmodernism : History, Theory, Fiction. New York: Routledge, 1988.

---. The Politics of Postmodernism. New York: Routledge, 2002.

INFELD, Leopold. Albert Einstein, His Work and Its Influence on Our World.

New York: Charles Scribner's Sons, 1950.

JAKI, Stanley. "The Absolute beneath the Relative: Reflections on Einstein's

Theories." Einstein and the Humanities. Ed. Ryan, Dennis P. New York:

Greenwood Press, 1987. 5-19.

JAMESON, Fredric. Postmodernism, or, the Cultural Logic of Late Capitalism. Durham: Duke University Press, 1991.

JOYCE, James. Finnegans Wake. London: Penguin Classics, 1999.

JUNG, C. G. The Archetypes and the Collective Unconscious. New York: Pantheon Books, 1959.

JUNG, C. G., and Mary FOOTE. The Visions Seminars : From the Complete Notes of Mary Foote. Zürich: Spring Publications, 1976.

LACAN, Jacques. Écrits. Paris: Éditions du Seuil, 1966.

---. De La Psychose Paranoïaque Dans Ses Rapports Avec La Personnalité Suivi De Premiers Écrits Sur La Paranoïa. Paris: Éditions du Seuil, 1975.

---. Autres Écrits. Paris: Editions du Seuil, 2001.

LYOTARD, Jean François. La Condition Postmoderne: Rapport Sur Le Savoir. Paris: Minuit, 1979.

NIETZSCHE, Friedrich. The Complete Works of Friedrich Nietzsche. New York: Gordon Press, 1974.

PALAHNIUK, Chuck. Fight Club. New York: Norton, 1996.

PYNCHON, Thomas. The Crying of Lot 49. Philadelphia: Lippincott, 1966.

REICHENBACH, Hans "The Philosophical Significance of the Theory of Relativity." Albert Einstein: Philosopher-Scientist. Ed. Schilpp, Paul Arthur. La Salle: Open Court, 1970. 269-87.

RULAND, Richard, y Malcolm BRADBURY. From Puritanism to Postmodernism: A History of American Literature. New York: Viking, 1991.

SCHRÖDINGER, Erwin. "Die Gegenwärtige Situation in Der Quantenmechanik." Die Naturwissenschaften, 2348 (1935): 844-49.

---. Science Theory and Man. New York: Dover Publications, 1957.

STROMBERG, Roland N. Makers of Modern Culture: Five Twentieth-Century Thinkers. Arlington Heights: Harlan Davidson, 1991. 
VONNEGUT, Kurt. Mother Night. New York: Delacorte Press, 1966.

---. Slanghterhouse-Five; or, the Children's Crusade, a Duty-Dance with Death. New York: Delacorte Press, 1969. 